\title{
Tear osmolarity changes after use of hydroxypropyl-guar-based lubricating eye drops
}

This article was published in the following Dove Press journal: Clinical Ophthalmology

\section{Alison $\mathrm{Ng}$ \\ Adam Keech \\ Lyndon Jones}

Centre for Ocular Research \& Education (CORE), School of Optometry \& Vision Science, University of Waterloo, Waterloo, ON, Canada
Correspondence: Alison $\mathrm{Ng}$ Centre for Ocular Research \& Education (CORE), School of Optometry \& Vision Science, University of Waterloo, 200 University Avenue West, Waterloo, ON, Canada N2L 3GI Fax +I 5198884303

Email alison.ng@uwaterloo.ca
Purpose: To evaluate tear osmolarity after using a hydroxypropyl-guar (HP-guar)-based lubricating eye drop four times daily (QID) for 3 weeks.

Methods: Thirty-one participants with dry eye disease (Ocular Surface Disease Index [OSDI] score $\geq 20$ and tear osmolarity $\geq 300 \mathrm{mOsm} / \mathrm{L}$ in at least one eye) were enrolled in this prospective, dispensing, non-randomized study involving a baseline visit and 3-week follow-up. Tear osmolarity, non-invasive tear break up time (NITBUT), conjunctival hyperemia, corneal and conjunctival staining were determined at baseline. Participants were instructed to instill one drop of a HP-guar-based drop QID in each eye for 3 weeks. At the follow-up visit, the symptoms and ocular surface parameters were reassessed. At this visit, one HP-guar drop was instilled into each eye and osmolarity was measured after 15 minutes, to examine short-term changes in osmolarity.

Results: Twenty-eight participants completed the study (5M, 23F; median age $54 \mathrm{yrs}$,

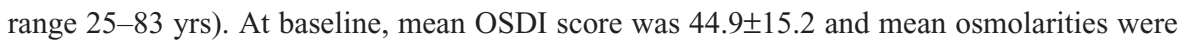
$314.63 \pm 11.9 / 306.6 \pm 10.1 \mathrm{mOsm} / \mathrm{L}$ (worst eye [WE]/better eye [BE]). After 3 weeks, mean osmolarity reduced to $307.7 \pm 15.7 / 303.9 \pm 11.3 \mathrm{mOsm} / \mathrm{L}$ (WE/BE; $p<0.05$ and $p=0.228$, respectively) and mean OSDI scores reduced to $28.3 \pm 17.0(p<0.01)$. A significant reduction in osmolarity was observed 15 minutes after instilling the lubricating drop ( $p<0.05 \mathrm{WE}, p=0.09 \mathrm{BE}$ ). Significant improvements in central corneal staining $(p<0.05$ OU) and NITBUT $(p<0.05$ WE only) were observed. Reductions in conjunctival staining and hyperemia were observed, but were not significant $(p>0.05)$.

Conclusions: A significant reduction in tear osmolarity and improvements in dry eye symptoms, corneal staining, and NITBUT were observed after 3 weeks of QID use of a HP-guarbased lubricant drop. A decrease in osmolarity was also demonstrated 15 minutes after drop instillation.

Keywords: eye drop, dry eye, osmolarity, tear film, HP-guar

\section{Introduction}

Tear film hyperosmolarity is a recognized hallmark of dry eye disease. Hyperosmolarity increases corneal and conjunctival epithelial stress, which activates a wide variety of inflammatory processes, involving mitogen-activated protein kinases and NF- $\kappa \mathrm{B}$ signaling. The hyperosmolar environment results in upregulation of HLA-DR (human leukocyte antigen - antigen D related), expression of proinflammatory cytokines, chemokines, and matrix metalloproteinases, epithelial cell death by apoptosis, and a loss in conjunctival goblet cells, leading to mucin-glycocalyx disruption. ${ }^{1}$ These factors perpetuate tear instability and subsequently increased tear evaporation, which, particularly in the aqueous-deficient patient, leads to further periods of tear hyperosmolarity until compensatory mechanisms are exhausted and osmolarity remains irreversibly 
in the pathological range. ${ }^{2}$ Exiting the inflammatory dry eye cycle involves targeting tear instability, ocular surface inflammation and addressing tear hyperosmolarity. ${ }^{3}$

Lubricating eye drops remain a common method of dry eye management to alleviate both signs and symptoms of the condition. The composition of ocular lubricants differ between products and common demulcents include hydroxypropyl methylcellulose, polyvinyl alcohol, carboxymethylcellulose, polyethylene glycol, and propylene glycol. ${ }^{4}$ Desirable features of lubricating eye drops include a long retention time on the ocular surface, in addition to addressing tear osmolarity.

Hydroxypropyl-guar (HP-guar) is an agent found in lubricating eye drops which has some unique characteristics. HP-guar acts as a viscous gelling agent and is derived from natural guar galactomannan, a water-soluble polysaccharide which has been treated with propylene oxide. ${ }^{5,6} \mathrm{HP}$-guar molecules have a high molecular weight (1,000-5,000 kDa), which accounts for the viscous nature of the gel. In a bottle, HP-guar, together with borate and sorbitol, form a loosely cross-linked, gel-like liquid, where sorbitol limits any further cross-linking. ${ }^{7}$ Upon instillation of the drop, the dilution of sorbitol in combination with the $\mathrm{pH}$ of the tear film causes the borate to interact with divalent ions (calcium, zinc, and magnesium) in the tear film to increase HP-guar crosslinking, forming a structured gel-like matrix over the ocular surface, in an attempt to prolong the retention of demulcents compared with other drops. ${ }^{7-9}$ Additionally, HP-guar molecules preferentially bind to desiccated or damaged hydrophobic regions of the cornea, allowing this protective layer to limit further damage and time for surface epithelial cells to undergo repair and renewal. ${ }^{5,8}$ The gel layer formed by the HP-guar network acts as a mucomimetic, compensating for mucin defects in the tear film, which also reduces the friction between the eyelid and ocular surface during blinks. ${ }^{9}$

A lubricating drop with a longer retention time and sustained lubrication is beneficial in interrupting the dry eye disease cycle in both aqueous-deficient and evaporative dry eye disease conditions, as both are driven by hyperosmolarity, despite originating from different causes. ${ }^{2}$ The addition of an eye drop should temporarily reduce tear osmolarity, may dilute any proinflammatory mediators on the ocular surface and provide a smooth, refractile surface. However, only sustained lubrication and adherence to the tear glycocalyx would protect the ocular surface from further epithelial insult and improve tear stability.

The purpose of this study was to evaluate tear osmolarity after using HP-guar lubricating eye drops with active ingredients polyethylene glycol 400 and polypropylene glycol (Systane ${ }^{\circledR}$ Ultra; Alcon Laboratories Inc., Fort Worth, TX, USA) four times daily for 3 weeks.

\section{Methods}

This study was conducted in accordance with Good Clinical Practice Guidelines and the tenets of the Declaration of Helsinki. All study procedures were conducted according to a protocol approved by the Office of Research Ethics at the University of Waterloo. Written informed consent was obtained from all participants before enrolment in the study.

This was a prospective, open-label, bilateral eye feasibility study. Thirty-one participants with signs and symptoms of dry eye disease were enrolled. Participants attended a screening visit to determine eligibility. A participant was eligible for inclusion if dry eye symptoms using the Ocular Surface Disease Index (OSDI, Allergan Inc., Irvine, CA, USA) questionnaire was $\geq 20$ and mean tear osmolarity, determined using the TearLab ${ }^{\mathrm{TM}}$ osmometer (TearLab Corporation, San Diego, CA, USA) was $\geq 300 \mathrm{mOsm} / \mathrm{L}$ in at least one eye. Exclusion criteria for this study were: any signs of other ocular surface disease; any systemic condition or use of any systemic or topical medication that may affect the ocular surface; any current use of Systane ${ }^{\circledR}$ or Systane ${ }^{\circledR}$ Ultra.

Eligible participants were dispensed HP-guar lubricating eye drops (Systane ${ }^{\circledR}$ Ultra, Alcon Laboratories Inc.) and instructed to instill one drop four times daily to each eye until the 3-week follow-up visit.

The following ocular surface parameters were evaluated at the baseline visit and the 3-week follow-up (Table 1):

- Tear osmolarity, assessed using the TearLab ${ }^{\mathrm{TM}}$ osmometer. The device was calibrated daily for quality control, using the supplied electronic check cards. Additionally, for each new box of test cards, the device was tested using TearLab ${ }^{\mathrm{TM}}$ normal and high osmolarity test solutions, used according to the manufacturer's instruction booklet.

Table I Ocular surface parameters

\begin{tabular}{ll}
\hline Parameter & Instrument/grading scale \\
\hline Tear osmolarity & $\begin{array}{l}\text { TearLab }^{\text {TM }} \text { osmometer } \\
\text { Placido rings (Eye Map, Alcon, Fort } \\
\text { Worth, TX) }\end{array}$ \\
Conjunctival hyperemia & $\begin{array}{l}\text { Alcon grading scale: } 0 \text { (white) }-3 \text { (marked } \\
\text { vascular dilation) }\end{array}$ \\
Corneal staining & Alcon grading scale: 0 (no staining) - 3 (severe \\
& macropunctate and coalesced staining) \\
Conjunctival staining & Alcon grading scale: 0 (no staining) - 3 (severe \\
& macropunctate and coalesced staining)
\end{tabular}

Abbreviation: NITBUT, non-invasive tear break-up time. 
A new TearLab test card was applied to a TearLab pen for each tear osmolarity measurement. For acquiring the tear osmolarity measurement, participants were instructed to look up and away from the investigator. The investigator gently placed the tip of the test card into the inferior tear meniscus, towards the outer canthus of the participant's eye, taking care to avoid any reflex tearing. Fifty nanolitres of tear fluid was taken up into the test card by capillary action, where successful sample collection was signaled by an audible beep. The pen and test card were docked onto the base unit, where the osmolarity value was displayed.

- Non-invasive tear break-up time (NITBUT), assessed using illuminated placido rings projected onto the tear film using the Eye Map topographer (Alcon Laboratories Inc.). For each measurement, participants were instructed to look straight ahead into the device, where a placido ring pattern was projected on their tear film. Participants were instructed to blink three times, and to hold their eyes open after the last blink until they were instructed to blink by the investigator (ie, the moment the first tear break-up was detected) or until the participant felt they were unable to hold their eyes open any longer. A stopwatch was used to time the duration from the last blink to the first distortion that appeared on the placido ring. The average of three measurements were taken for each eye at each visit.

- Conjunctival hyperemia, assessed using a slit lamp biomicroscope by examining each quadrant of the conjunctiva with diffuse white illumination. An Alcon grading scale was used to grade redness, where $0=$ white and $3=$ marked vascular dilation.

- Corneal staining, assessed 1 minute after instilling sodium fluorescein into the inferior fornix. Corneal staining was observed using a slit lamp biomicroscope under cobalt blue illumination and a Wratten 12 filter. An Alcon grading scale was used to grade staining, where $0=$ no staining and $3=$ severe macropunctate and coalesced staining.

- Conjunctival staining, examined using sodium fluorescein instilled from the assessment of corneal staining and observed using cobalt blue illumination and a Wratten 12 filter. Conjunctival staining was graded using the Alcon grading scale where $0=$ no staining and $3=$ severe macropunctate and coalesced staining.

At the follow-up visit, first the OSDI questionnaire and tear osmolarity were reassessed. To examine short-term changes of the eye drop on tear osmolarity, at the end of these assessments, one drop of HP-Guar eye drop was instilled into each eye and tear osmolarity was measured after 15 minutes. The intent to investigate the effect of the eye drop on short-term tear osmolarity was to observe how much of a reduction in tear osmolarity may occur after using the drop. To minimize the risk of measuring the osmolarity of the instilled drop itself, a wait-time of 15 minutes was selected as this was deemed a reasonable time point whereby any excess eye drop on the ocular surface would have cleared via normal tear turnover yet any anticipated dilution or reduction in tear osmolarity would be measurable.

After the second osmolarity measurements, the remaining ocular surface parameters were reassessed (Table 1).

Statistical analysis was conducted using SPSS Version 22 (IBM). Data were tested for normality using the ShapiroWilk test, where the alpha level 0.05 was set. As all data were normally distributed, parametric statistical tests were conducted. The worst eye (WE) is defined as the eye which had the greatest tear osmolarity, determined at baseline. Conversely, the better eye (BE) is defined as the eye which had the lowest tear osmolarity, determined at baseline.

\section{Results}

Thirty-one participants were enrolled in the study and 28 participants completed the study $(5 \mathrm{M} ; 23 \mathrm{~F}$ : median age 54 years, range $25-83$ years). Three participants were discontinued from the study for not maintaining the required study visits or for not following study procedures.

\section{Tear osmolarity}

At baseline, mean osmolarity in the WE was $314.63 \pm 11.9$ $\mathrm{mOsm} / \mathrm{L}$. After 3 weeks of drop use, mean osmolarity reduced to $307.7 \pm 15.7 \mathrm{mOsm} / \mathrm{L}$ in the WE $(p<0.05$; Figure 1). In the BE, the mean osmolarity at baseline was

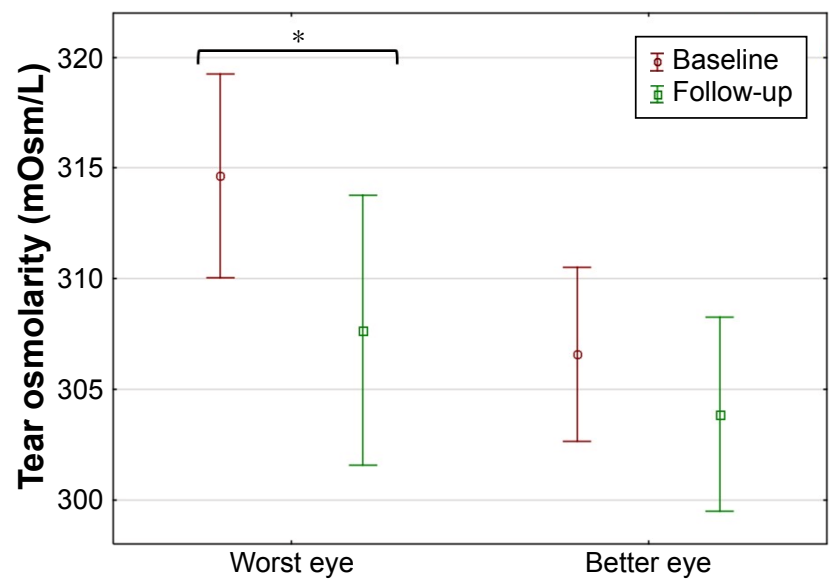

Figure I Tear osmolarity measured with the TearLab ${ }^{\mathrm{TM}}$ osmometer in the WE and $\mathrm{BE}$ at baseline and after 3 weeks of drop use (mean and $95 \% \mathrm{Cl}$ shown).

Note: A significant reduction in tear osmolarity was found after 3 weeks of drop use in the WE only $\left({ }^{*} p<0.05\right)$.

Abbreviations: WE, worst eye; BE, better eye. 
$306.6 \pm 10.1 \mathrm{mOsm} / \mathrm{L}$ and reduced to $303.9 \pm 11.3 \mathrm{mOsm} / \mathrm{L}$ after 3 weeks of drop use ( $p=0.228$ ).

At the follow-up visit, a significant reduction in osmolarity was observed 15 minutes after instilling the lubricating drop in the WE, from $307.7 \pm 15.7$ to $299.3 \pm 13.4 \mathrm{mOsm} / \mathrm{L}$ $(p<0.05)$ and a reduction from $303.9 \pm 11.3$ to $298.2 \pm 12.9$ $\mathrm{mOsm} / \mathrm{L}$ in the BE approached significance $(p=0.09$, Figure 2).

\section{Symptoms}

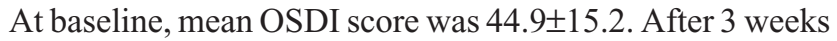
of drop use, the mean OSDI score significantly reduced to $28.3 \pm 17.0(p<0.01)$.

\section{Clinical signs}

There were no changes in high or low contrast visual acuity between visits (all $p>0.05$ ).

The mean NITBUT at baseline was $4.5 \pm 2.9$ and $5.0 \pm 2.3$ seconds (WE and BE, respectively), and after 3 weeks of drop use improved to $6.7 \pm 4.6$ and $5.6 \pm 3.6$ seconds. There was a statistically significant improvement in the WE only (WE $p<0.05$, BE $p=0.46$; Figure 3).

Improvements in corneal staining were observed in all quadrants in both eyes at the follow-up visit. However, a significant improvement was observed only in the central cornea in both eyes $(p<0.00001$, Figure 4$)$.

Small reductions in conjunctival staining scores and conjunctival hyperemia in both eyes were observed, but these changes were not significant $(p>0.05)$.

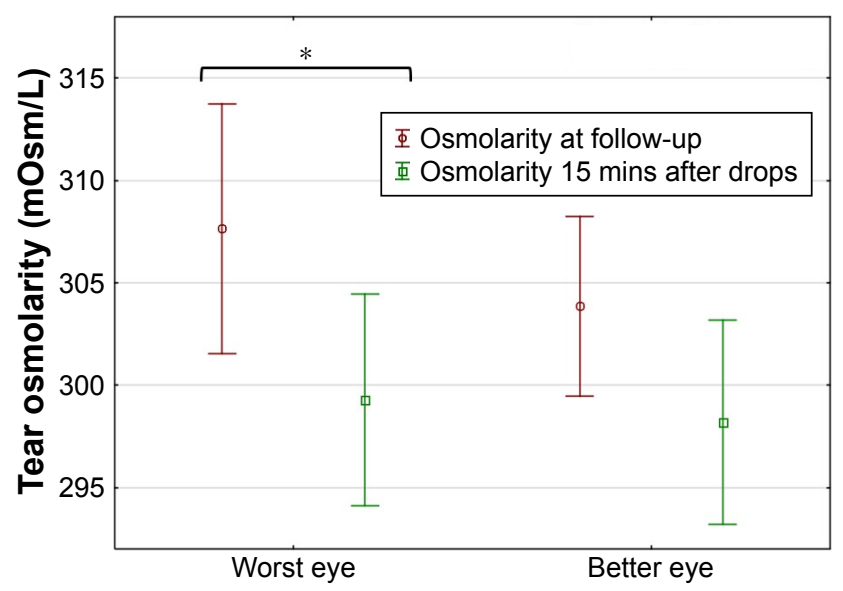

Figure 2 Tear osmolarity measured with the TearLab ${ }^{\mathrm{TM}}$ osmometer in the WE and $\mathrm{BE}$ before and 15 minutes after instillation of HP-guar drop, determined at the follow-up visit (mean and $95 \% \mathrm{Cl}$ shown).

Note: A significant reduction in tear osmolarity 15 minutes after instillation of the drop in the WE only $\left({ }^{*} p<0.05\right)$.

Abbreviations: WE, worst eye; BE, better eye.

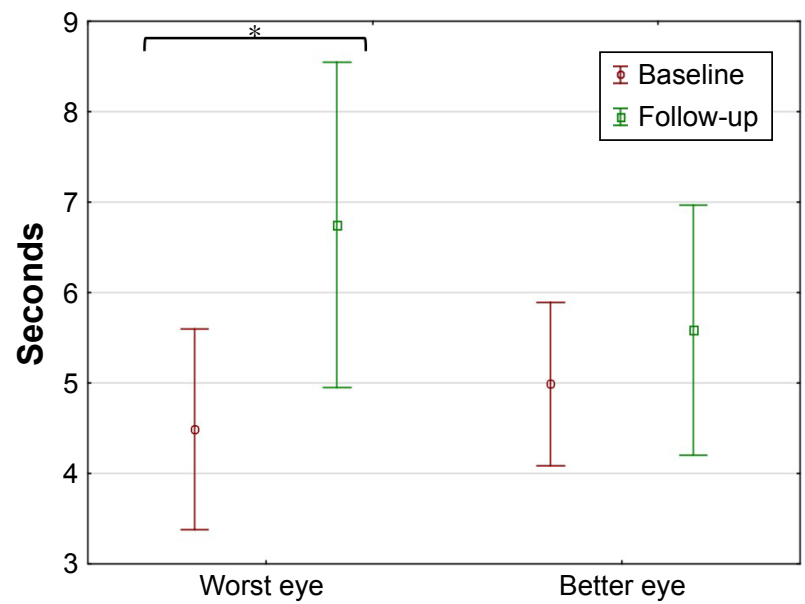

Figure 3 Non-invasive tear break-up time at baseline and after 3 weeks of drop use (mean and $95 \% \mathrm{Cl}$ shown).

Note: A significant improvement in stability was found after 3 weeks of drop use in the WE only $(* p<0.05)$.

Abbreviation: WE, worst eye.

\section{Discussion}

Lubricating eye drops remain the main treatment option for patients with dry eye disease. This study has shown that in a group of dry eye participants (determined by tear osmolarity) that were compliant in a fixed regimen of HP-guar eye drops, tear osmolarity was reduced and significant improvements in symptoms, tears stability and corneal staining were found after only 3 weeks of drop use.

The impact of tear hyperosmolarity is multifactorial and cyclical in nature. Hyperosmolarity leads to a cycle of inflammation, ocular surface damage and discomfort, which

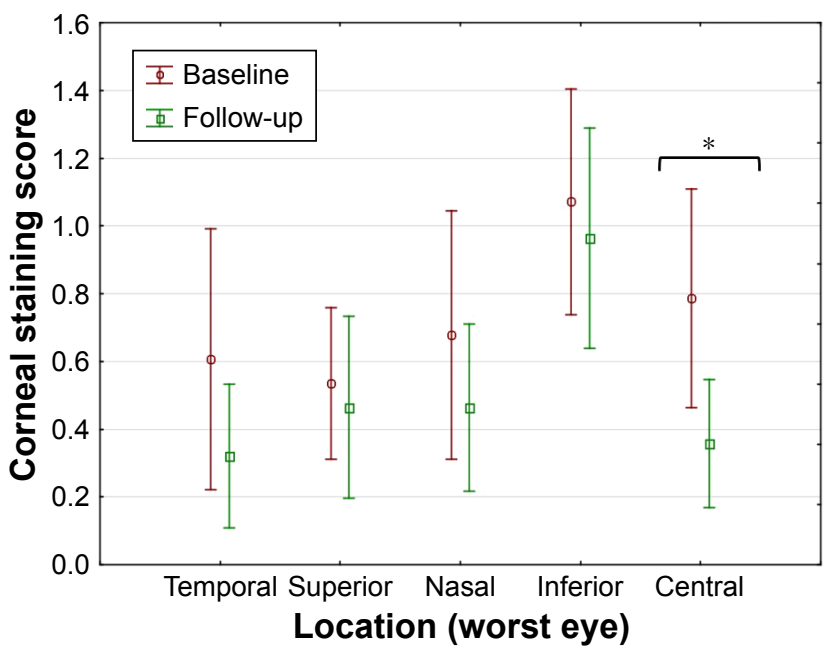

Figure 4 Corneal staining at baseline and after 3 weeks of drop use (WE, mean and $95 \% \mathrm{Cl}$ shown).

Note: A significant improvement in corneal staining was found after 3 weeks of drop use in the central region of the WE only $\left({ }^{*} p<0.01\right)$.

Abbreviation: WE, worst eye. 
is commonly observed in dry eye. ${ }^{2,10}$ Disrupting this cycle by supplementing the aqueous phase by instilling an eye drop, will transiently reduce hyperosmolarity, as demonstrated in this study, amongst others. ${ }^{11-13}$ Several previous studies have shown that artificial tears can be used to reduce tear osmolarity. ${ }^{11-13}$ While hypotonic drops may reduce tear osmolarity for only 1-2 minutes, ${ }^{3}$ this study found a reduction in tear osmolarity still remains some 15 minutes after drop instillation. To our knowledge, this is the only study to have determined the time after which such a drop in osmolarity remains.

Most notably, in this study, the reduction in tear osmolarity after 3 weeks of drop use was greatest in the WE (ie, the eye with the greatest tear osmolarity at baseline). The improvements in the WEs also showed the greatest amount of improvement in NITBUT and central corneal staining. While the BEs also demonstrated reductions in osmolarity and improved NITBUT, these were small and did not reach significance. The improvements in osmolarity and tear stability after 3 weeks of drop use are important findings in light of the cyclical damage that hyperosmolarity can cause. Improvements in both of these metrics reduces corneal epithelial cell damage, indicated by the reduced levels of corneal staining in all quadrants at the follow-up visit. Although this study did not find clinically significant changes in conjunctival staining with HP-guar drop use, a previous study did find a significant reduction in conjunctival staining compared with a control drop. ${ }^{5}$

The HP-guar drop used in this study contains two hydrogel polymer demulcents $(0.4 \%$ polyethylene glycol 400 and $0.3 \%$ propylene glycol). Upon instillation, HP-guar crosslinking occurs to form a structured gel-like network over the ocular surface to prolong the retention of demulcents. ${ }^{7,8}$ This protective layer preferentially binds to desiccated or damaged hydrophobic regions of the cornea ${ }^{5}$ and also acts as a mucomimetic, reducing the friction between the eyelid and ocular surface during blinks. ${ }^{9}$

The hydrophilic mucins expressed within the tear film are responsible for stabilizing the tear film, decreasing the surface tension such that the tear film can readily spread over the ocular surface. Tear hyperosmolarity is a contributing factor to goblet cell loss, ${ }^{3}$ a characteristic of dry eye disease, which disrupts the production of gel-like mucins., ${ }^{3,10}$ Moon et al found artificial tears containing HP-guar significantly increased the thickness of the mucous layer of the tear film for up to 30 minutes after instillation compared to a control eye drop. ${ }^{14}$ Using a HP-guar-based eye drop is likely to improve adherence and retention of the artificial tear product, improving tear stability and minimizing tear evaporation, thereby lowering tear osmolarity and corneal desiccation and improving symptoms, potentially offering longer lasting benefits to the dry eye patient.

In this study, participants were instructed to use the HPguar eye drop four times daily for 3 weeks. Chronic exposure of the ocular surface to preservatives such as benzalkonium chloride (BAK) is now well established to induce toxicity and adverse changes to the ocular surface in patients with dry eye disease ${ }^{15-17}$ Extensive evidence exists to confirm that patients with severe dry eye disease who require frequent dosing with lubricants should avoid the use of ocular lubricants preserved with BAK. Milder preservative systems, such as polyquaternium-1 (Polyquad ${ }^{\circledR}$, Alcon Laboratories Inc., Fort Worth, TX, USA), found in the HP-guar-based drop used in this study, are more tolerable and milder to the ocular surface than BAK. ${ }^{18}$ While the duration of this feasibility study was short, the impact of drops preserved with Polyquad ${ }^{\circledR}$ versus unpreserved, single-use vials of the HP-guar-based eye drops would be a further study worth considering in the long-term management of patients with dry eye.

As this was a feasibility study, no control eye drop was selected. Further studies would benefit from utilizing a control eye drop and a randomized control, masked study design.

In conclusion, the results from this study indicate a significant decrease in tear osmolarity following a regimen of four-times-daily instillation of HP-guar eye drops for 3 weeks. In addition, such a regimen was effective in lowering tear osmolarity, improving dry eye symptoms and ocular surface measures such as tear stability and corneal staining. Determination of the osmolarity post-instillation also confirmed that there was a significant reduction 15 minutes after instilling the HP-guar lubricating eye drops.

\section{Acknowledgments}

This work was presented as a poster at the Association for Research in Vision and Ophthalmology Annual Meeting, Denver, Colorado, May 2015.

This study was funded by Alcon Laboratories, Inc., Fort Worth, TX, USA.

\section{Disclosure}

CORE employees have received funds for conducting research from the following: Advanced Vision Research, Alcon, AlgiPharma, Allergan, CIBA Vision, CooperVision, Essilor, Johnson \& Johnson Vision Care, Ocular Dynamics, 
Oculus, Ocusense, TearScience, and Visioneering Technologies. LJ is a consultant and/or serves on an advisory board for Alcon and J\&J Vision Care. The authors report no other conflicts of interest in this work.

\section{References}

1. Bron AJ, de Paiva CS, Chauhan SK, et al. TFOS DEWS II pathophysiology report. Ocul Surf. 2017;15(3):438-510.

2. Bron AJ, Yokoi N, Gafney E, Tiffany JM. Predicted phenotypes of dry eye: proposed consequences of its natural history. Ocul Surf. 2009; 7(2):78-92.

3. Baudouin C, Aragona P, Messmer EM, et al. Role of hyperosmolarity in the pathogenesis and management of dry eye disease: proceedings of the OCEAN group meeting. Ocul Surf. 2013;11(4):246-258.

4. Tong L, Petznick A, Lee S, Tan J. Choice of artificial tear formulation for patients with dry eye: where do we start? Cornea. 2012;31 Suppl 1: S32-S36.

5. Christensen MT, Cohen S, Rinehart J, et al. Clinical evaluation of an HP-guar gellable lubricant eye drop for the relief of dryness of the eye. Curr Eye Res. 2004;28(1):55-62.

6. Cheng Y, Brown KM, Prud'homme RK. Characterization and intermolecular interactions of hydroxypropyl guar solutions. Biomacromolecules. 2002;3(3):456-461.

7. Springs CL. Novel hydroxypropyl-guar gellable lubricant eye drops for treatment of dry eye. Adv Ther. 2010;27(10):681-690.

8. Benelli U. Systane lubricant eye drops in the management of ocular dryness. Clin Ophthalmol. 2011;5:783-790.
9. Petricek I, Berta A, Higazy MT, Németh J, Prost ME. Hydroxypropylguar gellable lubricant eye drops for dry eye treatment. Expert Opin Pharmacother. 2008;9(8):1431-1436.

10. Schaumberg DA, Gulati A, Mathers WD, et al. Development and validation of a short global dry eye symptom index. Ocul Surf. 2007;5(1): $50-57$.

11. Cömez AT, Tufan HA, Kocabiyik O, Gencer B. Effects of lubricating agents with different osmolalities on tear osmolarity and other tear function tests in patients with dry eye. Curr Eye Res. 2013;38(11): 1095-1103.

12. Benelli U, Nardi M, Posarelli C, Albert TG. Tear osmolarity measurement using the TearLab Osmolarity System in the assessment of dry eye treatment effectiveness. Cont Lens Anterior Eye. 2010;33(2):61-67.

13. McCann LC, Tomlinson A, Pearce EI, Papa V. Effectiveness of artificial tears in the management of evaporative dry eye. Cornea. 2012; 31(1): $1-5$.

14. Moon SW, Hwang JH, Chung SH, Nam KH. The impact of artificial tears containing hydroxypropyl guar on mucous layer. Cornea. 2010; 29(12):1430-1435.

15. Noecker R. Effects of common ophthalmic preservatives on ocular health. Adv Ther. 2001;18(5):205-215.

16. Stewart WC, Stewart JA, Nelson LA. Ocular surface disease in patients with ocular hypertension and glaucoma. Curr Eye Res. 2011;36(5): 391-398.

17. Stalmans I, Sunaric Mégevand G, Cordeiro MF, et al. Preservative-free treatment in glaucoma: who, when, and why. Eur J Ophthalmol. 2013; 23(4):518-525.

18. Rolando M, Crider JY, Kahook MY. Ophthalmic preservatives: focus on polyquaternium-1. Expert Opin Drug Deliv. 2011;8(11):1425-1438.
Clinical Ophthalmology

\section{Publish your work in this journal}

Clinical Ophthalmology is an international, peer-reviewed journal covering all subspecialties within ophthalmology. Key topics include: Optometry; Visual science; Pharmacology and drug therapy in eye diseases; Basic Sciences; Primary and Secondary eye care; Patient Safety and Quality of Care Improvements. This journal is indexed on

\section{Dovepress}

PubMed Central and CAS, and is the official journal of The Society of Clinical Ophthalmology (SCO). The manuscript management system is completely online and includes a very quick and fair peer-review system, which is all easy to use. Visit http://www.dovepress.com/ testimonials.php to read real quotes from published authors. 